\title{
A review of acoustic aspect in synthetic jet
}

\author{
Mikołaj Szyca ${ }^{1}$ and Emil Smyk ${ }^{1 *}$ \\ ${ }^{1}$ Faculty of Mechanical Engineering, Bydgoszcz University of Science and Technology, \\ al. Prof. S. Kaliskiego 7, 85-796 Bydgoszcz, Poland
}

\begin{abstract}
The synthetic jet actuators are promising heat transfer enhancement devices. They are used in active cooling systems and the future may replace classic fan systems. Although the flow and the heat transfer parameters are very important, these devices must be quiet enough to be used in offices and other spaces. In this paper, the acoustic investigations of the synthetic jet are compiled and discussed. The impact of actuators design on generated noise and the possible ways to noise decreasing are presented. In the end, the list of gaps and challenges are presented for laying down the foundation for future research.
\end{abstract}

\section{Introduction}

The synthetic jet (SJ) is generated by periodic injection and expulsion of some volume of fluid into the closed cavity. The expelled part of fluid moves away from the orifice and around them the edge vortices are generated - synthesized. Then, the new volume of fluid from the surroundings is injected into the cavity. The net mass flow in the orifice of the synthetic jet actuator (SJA) is zero, and therefore this jet is called zero-net mass-flux (ZNMF). The change of the volume of the cavity can be realized by the replace one [1] or more [2] walls of the actuator with the movable or deformable element as piezoelectrics $[3,4]$, loudspeaker $[5,6]$, or piston [7]. The schematic presentation of synthetic jet generation is presented in Fig. 1.

a)

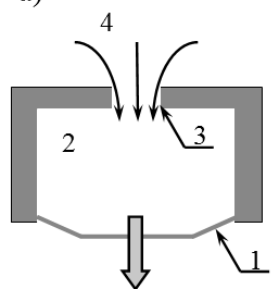

b)

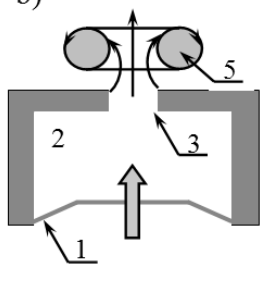

c)

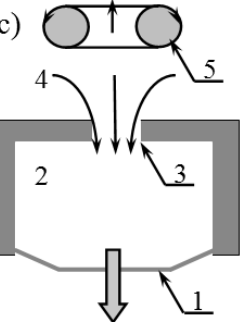

Fig. 1. Synthetic jet actuator a) the suction phase; b) the injection phase; c) the suction phase and the moving away of the synthetic jet (1 - diaphragm; 2 - cavity; 3 - nozzle; 4 - jet sucked in from the environment; 5 - edge vortices)

The turbulent nature of the SJ made that they found many applications. They are used for the mixing enhancement $[8,9]$, heat transfer enhancement $[1,10,11]$, active flow control [1214], and underwater propulsion systems [15-17]. With such a wide range of applications,

${ }^{*}$ Corresponding author: emil.smyk@pbs.edu.pl 
many parameters of SJ are investigated and should be taken into account during the actuator design. For this reason, there are more and more review articles devoted to a specific aspect of SJ, as LEM modeling [18], the impact of geometric parameters on SJ properties [19], or due to the SJ application, like heat transfer enhancement [20-22] or other [23,24]. The number of papers about the SJA used in the cooling systems shows that this area is expressly interesting for researchers.

The SJs are used mainly as impinging jets in devices dedicated to electronics cooling $[25,26]$. An often overlooked aspect is the sound pressure level (SPL) generated by SJA. While the noise in the workspace is defined by the standards, eg. EN ISO 9241-6:2002 [27], and therefore the possibility of the SJA application is limited by the SPL generated by this device.

For this reason in dis paper, we want to present the knowledge about the acoustic aspect of the SJ. Because the number of articles in this area is not large, we referred to each article that we managed to find on this topic. We used the Web of Science, GoogleShoolar, ReaserchGate, and Scopus databases for the search of the article.

\section{Methodology of acoustic measurements}

Table 1 was presented the all-found article, in which the SPL of the SJA was measured. The year and the methodology of the measurements were also presented.

Table 1. Measured values.

\begin{tabular}{|c|c|c|c|}
\hline Article & Year & Used method & $\begin{array}{c}\text { Actuator } \\
\text { type }\end{array}$ \\
\hline Arik, M [28] & 2007 & Anechoic chamber & Piezoelectric \\
\hline Lasance et al. [29] & 2008 & $\begin{array}{l}\text { Reverberant chamber } \\
\text { (ISO 3741:1999) }\end{array}$ & Acoustic \\
\hline Bhapkar et al. [30] & 2013 & $\begin{array}{l}\text { Chamber without } \\
\text { other sound sources }\end{array}$ & Acoustic \\
\hline Bhapkar et al. [31] & 2014 & Room(ISO 3746:2010) & Acoustic \\
\hline Mangate and Chaudhari [32] & 2015 & $\begin{array}{l}\text { Anechoic chamber } \\
\text { (ISO 3746:2010) }\end{array}$ & Piezoelectric \\
\hline Jabbala and Jeyalingam [33] & 2017 & $\begin{array}{l}\text { Anechoic chamber } \\
\text { (ISO 3746:2010) }\end{array}$ & Piezoelectric \\
\hline Kanase et al. [6] & 2018 & $\begin{array}{l}\text { Anechoic chamber } \\
\text { (ISO 3746:2010) }\end{array}$ & Piezoelectric \\
\hline Jeyalingam and Jabbala[34] & 2018 & Semianechoic chamber & Piezoelectric \\
\hline Smyk et al. [35] & 2020 & Room(ISO 3746:2010) & Acoustic \\
\hline Smyk et al. [36] & 2021 & Room(ISO 3746:2010) & Acoustic \\
\hline Smyk and Markowicz[37] & 2021 & Room(ISO 3746:2010) & Acoustic \\
\hline Smyk $[38]$ & 2021 & Room(ISO 3746:2010) & Acoustic \\
\hline Gil et al. [39] & 2021 & Room(ISO 3746:2010) & Acoustic \\
\hline Ikhlaq et al. [40] & 2021 & 3 microphones around SJA & Piezoelectric \\
\hline
\end{tabular}

Most papers used the ISO 3746:2010 [41] as a standard for the noise measurements. It means that the SPL measurements were made in an anechoic chamber or in the room where the SPL of the environment was at least $10 \mathrm{~dB}$ lower than the SPL generated by the SJA. The methodology of the noise measurements was correctly described. 


\section{The noise level in an SJA}

Lasance et al. [29] compared the noise generated by the acoustic SJA to the noise generated by the fan. Both devices were used for the flat plate cooling and at the same driving power the SJA was quieter and more effective than the fan. The noise level was dependent on the geometric parameters of the actuator body. Therefore in this section, the impact of the chosen parameters of synthetic jet on the generated noise was described. The chosen noise level was given to enable the comparison of the results to the other devices.

The SPL generated by the actuator strongly depends on the driving frequency and actuator driving power. For example in the case investigated by Ikhlak et al. [40], the maximum SPL range was from 48 to $97 \mathrm{~dB}$, and in the case showed by Smyk [38] the SPL was in the range from 49 to $58 \mathrm{~dB}$ at the power from 5 to $12 \mathrm{~W}$. However, supply parameters are easy to modify and have also impact on the flow parameters of SJ. Therefore in this paper will be taken into account the only impact of the geometrical parameters on the generated noise. These parameters can not be modified on the fly and must be selected at the design stage of the actuator.

\subsection{The impact of the orifice diameter}

The diameter of the orifice has an impact on many SJ parameters, also on the noise generated by the actuator. Kanase et al. [6] investigate the SPL as a function of acoustic Reynolds number for different orifice parameters. They showed that the noise generated by the SJA is inversely proportional to the orifice diameter. For example, the actuator with the orifice diameter $d=8 \mathrm{~mm}$ generated the SPL $=68 \mathrm{~dB}$ and at $d=5 \mathrm{~mm}$ generated the SPL $=65 \mathrm{~dB}$, at the same driving power and the orifice length.

This dependence was not confirmed in another paper [29,30]. Lasance et al. [29] showed that the smaller diameter the quieter actuator. However, the differences are caused also by the orifice length to the orifice diameter ratio (aspect ratio, $A R=l / d$ ). The difference between the SPL generated by the actuator with orifice diameter $d=3 \mathrm{~mm}$ and $d=4 \mathrm{~mm}$ is equal to $3,3 \mathrm{~dB}$ at the orifice length $l=30 \mathrm{~mm}, 4,9 \mathrm{~dB}$ at the orifice length $l=90 \mathrm{~mm}$, and $12,1 \mathrm{~dB}$ at the orifice length $l=120 \mathrm{~mm}$ (the power was not the same in these cases).

A similar conclusion was presented by Bhapkar et al.[30]. The smaller diameter the quieter actuator. Bhapkar et al.[30] suggest that the noise is related to the volume of the air oscillation in the orifice. On the other hand, the decreasing of the orifice diameter caused the increase in the SJ velocity and turbulence of the jet, which is correlated with the noise generation[42].

The results presented by Gil et al. [39] for the multiple-orifice SJA confirmed results presented by Kanase et al. [6] and showed that the lower velocity in the orifices the lower the noise level. However, the SPL has a local maximum, that is correlated with the optimal diameter of SJ [43]. At this point, the velocity is maximal and the SPL is the highest. This is in line with what is known about the relationship between turbulence and noise[42].

Based on the presented results and based on the methodology of these papers it must be said that the SPL is inversely proportional to the orifice diameter. The measurements in both papers [6,39] were made according to the standard ISO 3746:2010 [41] and the results are unambiguous and consistent with other papers.

\subsection{The impact of the orifice length}

Already Lasance et al. [29] showed that the SPL depends on the orifice length. It was confirmed by Kanase et al. [6]. The measurements were made by the actuators with the same diameter $(d=3,5,8,12,14)$ and different orifice length $(l=3,5,8,12)$. The results were 
compared for the same diameter and different aspect ratio and in all cases the lower aspect ratio the higher SPL. It means that the noise is higher, the shorter is the orifice. It is caused by the decrease of acoustic energy due to multiple reflections in the orifice cavity.

Bhapkar et al. [31] obtained a reverse dependency. They compared the actuators with different orifice diameters and lengths but with the same aspect ratio. Based on the research presented earlier, it should be noted that Bhapkar et al. [31] made a mistake of inference during their research. While maintaining the same aspect ratio, they assessed that it is the length of the orifice that impacts the generated SPL, and not the orifice diameter, which in this case turned out to be the decisive factor.

It should therefore be said that the SPL generated by SJA is inversely proportional to the orifice length.

\subsection{The impact of the orifice shape}

The shape of the SJA orifice is one of the most frequently studied parameters. Although circle and slot orifices are usually investigated, orifices with other shapes are also tested.

Mangate and Chaudhari [32] investigated the actuator with a circular, diamond, and oval cross-sectional shape orifice. They showed that the highest SPL was generated by the actuator with a round nozzle. This noise was even $5 \mathrm{~dB}$ higher than in the case of an actuator with an oval orifice. The diamond orifice generated the lowest noise level at low acoustic Reynolds numbers ( $R e \leq 6,000$, a difference from 1 to $5 \mathrm{~dB}$ compared to other orifices shapes). At acoustic Reynolds number greater than 6,000 , the actuator with oval orifice generated the lowest SPL. In the case of $R e=10,000$, an actuator with an oval orifice generated noise $8 \mathrm{~dB}$ lower than that of an actuator with a diamond orifice, and $13 \mathrm{~dB}$ lower than the actuator with a round orifice.

Bhapkar et al. [31] investigated the SJA with elliptical, slot, circular, and square orifice for the same hydraulic diameter $(d=12 \mathrm{~mm})$. Measurements were made for 4 different frequencies. The results were not clear. It should be noted that the tested actuators had different resonance frequencies and the noise generated at the resonant frequency was always lower by $8-12 \mathrm{~dB}$ than the SPL for other frequencies. A similar relationship was noted by Kanase et al. [6].

The other way was proposed by Smyk et al. [36]. They compared the SPL generated by the actuator with circular, slot, and square orifices. The compared orifices do have not the same aspect ratio but with the same area. The length of the orifice was equal to the characteristic dimension of the orifice. The highest SPL was generated by the actuator with the slot orifice and the smaller by the actuator with the square orifices. The differences were significant and equal to about $8 \mathrm{~dB}$ (between the clot and rectangle orifice).

Base on these results, it should be said that the shape of the orifice has a significant effect on the SPL generated by the actuator. The circular orifice has one of the worst impacts on the generated noise. Despite the fact, the slot orifice is commonly used in research still the impact of the slot orifice aspect ratio (height to length ratio) on the SPL was not investigated.

\subsubsection{The chevron orifice}

The use of the chevron orifice or nozzle can reduce the noise generated by the jet $[44,45]$. Smyk and Markowicz [37] and Smyk [38] investigated the impact of the chevron orifice on the SPL generated by the SJ. They showed that the chevron orifice depending on the number of chevrons and their height can reduce the SPL. The results were not unambiguous and it is not possible to decide when the chevron orifice reduces or increases the generated noise, based on theirs. The SPL differences between the classic circular orifices and chevron orifices were small and not bigger than $1.5 \mathrm{~dB}$. Bur in cases, when chevron orifices increase the SPL 
the difference was bigger and was even equal to $3 \mathrm{~dB}$. Although the chevron nozzle was used in SJA in $[46,47]$ the impact of the chevron nozzle on acoustic parameters of SJ was not investigated. We can only try to interpolate the results obtained for the continuous jet on the SJ.

\subsection{The other parameters}

The impact of the other geometric parameters as a cavity shape or a cavity volume still was not investigated. The significant impact on the SPL has an element used to the SJ generation (piezoelectric, loudspeaker, piston). Smyk et al. [35] compared woofer and mid-range driver with a similar specification. The noise generated by the actuator with the mid-range driver was about $10-14 \mathrm{~dB}$ higher than in the case of the actuator with the woofer. Additionally, they used the multiple-orifice actuator body with or without fins in the cavity (Fig. 2). The lowest SPL was generated by the actuator with classic fins in the cavity. It is one of the many special designs of SJ actuators and very difficult to use such results for other actuators.

(a)

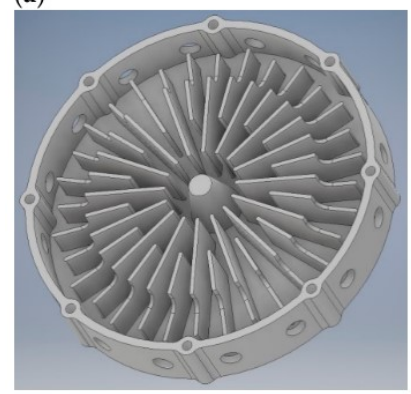

(b)

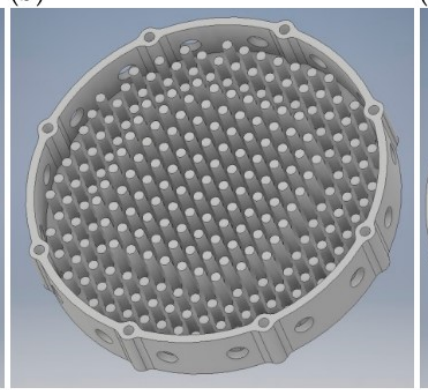

(c)

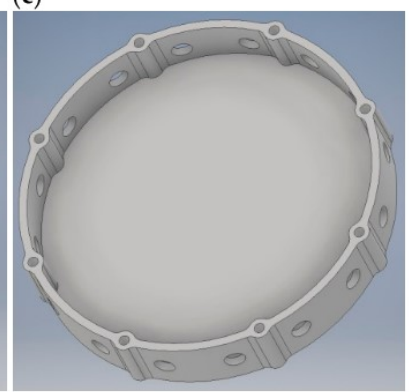

Fig. 2. The actuator body with (a, b) and without (c) fins in the cavity [35].

Jabbal and Jeyalingam [33] investigated the twin and classic SJA with the circular and lobed orifice. The classic oscillator (with one orifice) was made by the closing of one orifice. The actuators with one orifice generated a higher SPL than the twin actuator. The authors suggest that this is related to the anti-phase effect - in the case of two nozzles, the sound waves are phase-shifted by about $180^{\circ}$ and overlap each other, reducing the SPL.

Additionally, the actuator with the lobbed orifice was noisier by high frequency (above $2,000 \mathrm{~Hz}$ ), and by low frequencies, the actuator with circular orifices generated lower SPL. Similarly, as in the case of chevron orifice, the shape of the orifice was chosen because this shape is used in aircraft.

Arik [28] investigated the piezoelectric actuator generating the SPL in a range of $60-70 \mathrm{~dB}$. Therefore, to reduce noise, the researcher placed the generator in a small cube with a side length of 3 inches. On one of the sides of the cube, there is a small silencer connected by a $10 \mathrm{~mm}$ diameter hole with the chamber, and with a $1 ; 2$; or $3 \mathrm{~mm}$ hole on the opposite wall. The opening allowed free airflow between the atmosphere and the interior of the silencer and the chamber.

Arik [28] showed that the damping efficiency decreases with the increase of the orifice diameter in the muffler. Moreover, the most advantageous length of the silencer was indicated, which was around $2 \mathrm{~cm}$. It allowed reducing the noise from $65 \mathrm{~dB}$ to $25 \mathrm{~dB}$. Although the result is really impressive, it should be noted that this solution can be impossible or very difficult to use in practical application (in the cooling system), because the generators would have to be closed together with the object from which they receive heat or in which they perform the function of active flow control. Additionally in this solution, the heat should be removed from the cube. 


\section{Conclusion}

The paper presents a review of the papers that investigated the SPL generated by the SJA. The discussed articles showed that:

- the SPL is directly proportional to the supply power;

- the SPL is inversely proportional to the orifice diameter;

- the SPL is inversely proportional to the orifice length;

- the shape of the nozzle has a significant impact on the level of generated sound, and the SJA with a circular cross-section orifice generates the highest SPL;

- the lowest SPL generates the actuator with a square orifice, at the same crosssection orifice area;

- the chevron orifice can reduce the SPL generated by the actuator;

- the lobed orifice reduces the SPL generated by the actuator at high operation frequencies;

- the twin SJA generated lover SPL than single-orifice SJA.

There is still a lack of comprehensive investigation of the acoustic aspects of SJA. Among the biggest shortcomings and challenges that should be mentioned are:

- the investigations of the impact of the aspect ratio of slot orifice on the SPL;

- determining how much orifice diameter and length affect the SPL, so find the one relationship between aspect ratio and SPL;

- the investigation of nonclassic SJA, as hybrid SJ, multiple-orifice synthetic jet, etc.

- the investigation of the impact of cavity shape and volume on the SPL.

It must be noted, that even though the number of studies on SPL generated by SJ still increases this number is still very low. Although noise measurements are relatively simple and undemanding and can be performed additionally without affecting the main assumptions of the other tests.

\section{Acknowledgment}

This work was supported by the National Center for Research and Development, Poland. Grant No.: LIDER/6/0024/L-10/18/NCBR/2019.

\section{References}

1. P. Gil, Int. J. Therm. Sci. 166, 106949 (2021)

2. E. Smyk, S. Wawrzyniak, and K. Peszyński, Mech. Mech. Eng. 24, 17 (2020)

3. T. Yeom, L. Huang, M. Zhang, T. Simon, and T. Cui, Int. J. Heat Mass Transf. 143, 118484 (2019)

4. Y. H. Liu, S. Y. Tsai, and C. C. Wang, Appl. Therm. Eng. 75, 289 (2015)

5. P. Gil, J. Wilk, and M. Korzeniowski, Appl. Sci. 11, 5666 (2021)

6. M. M. Kanase, L. D. Mangate, and M. B. Chaudhari, J. Low Freq. Noise Vib. Act. Control 37, $31(2018)$

7. F. Xu, Z. Gao, X. Ming, L. Xia, Y. Wang, W. Sun, and R. Ma, Exp. Therm. Fluid Sci. 64, $94(2015)$

8. P. Wang and C. Shen, J. Zhejiang Univ. Sci. A 20, 701 (2019)

9. Q. Xia and S. Zhong, Flow Meas. Instrum. 53, 308 (2017)

10. P. Gil, E3S Web Conf. 100, 00017 (2019)

11. P. K. Singh, S. K. Sahu, P. K. Upadhyay, and S. Singh, Exp. Heat Transf. 00, 1 (2021)

12. Z. Hao, G. Liu, W. Ren, Y. Wang, and H. Bie, J. Appl. Fluid Mech. 14, 1053 (2021) 
13. P. Salunkhe, Y. Wu, and H. Tang, J. Fluids Eng. Trans. ASME 142, 1 (2020)

14. A. Mahboubidoust, A. Ramiar, and M. Dardel, Theor. Appl. Mech. Lett. 7, 185 (2017)

15. L. Geng, Z. Hu, and Y. Lin, Ocean Eng. 176, 84 (2019)

16. C. Tang, W. Ma, B. Li, M. Jin, and H. Chen, Adv. Eng. Mater. 1901130, 1 (2019)

17. M. Krieg and K. Mohseni, IEEE J. Ocean. Eng. 33, 123 (2008)

18. M. Chiatto, F. Capuano, G. Coppola, and L. De Luca, Sensors 17, 1216 (2017)

19. M. H. Hong, S. Y. Cheng, and S. Zhong, Phys. Fluids 32, 031301 (2020)

20. A. H. Ariffin and K. A. Ahmad, J. Adv. Res. Fluid Mech. Therm. Sci. 72, 103 (2020)

21. A. Arshad, M. Jabbal, and Y. Yan, Int. J. Heat Mass Transf. 146, 118815 (2020)

22. M. Ikhlaq, M. Yasir, M. Demiroplu, and M. Arik, in IEEE Trans. Components, Packag. Manuf. Technol. (2021), pp. 1156-1170

23. P. Sharma, P. K. Singh, S. K. Sahu, and H. Yadav, A Critical Review on Flow and Heat Transfer Characteristics of Synthetic Jet (Springer Singapore, 2021)

24. H. Zong, M. Chiatto, M. Kotsonis, and L. de Luca, Actuators 7, 77 (2018)

25. R. Mahalingam, Annu. IEEE Semicond. Therm. Meas. Manag. Symp. 196 (2007)

26. P. K. Singh, S. K. Sahu, and P. K. Upadhyay, Exp. Heat Transf. 1 (2020)

27. International Organization for Standardization (ISO), EN ISO 9241-6:2002. Ergonomic Requirements for Office Work with Visual Display Terminals (VDTs) -Part 6: Guidance on the Work Environment (ISO 9241-6:1999) (ISO: Geneva, Switzerland, 1999), p. 32

28. M. Arik, Appl. Therm. Eng. 27, 1483 (2007)

29. C. J. M. Lasance, R. M. Aarts, and O. Ouweltjes, in 2008 Twenty-Fourth Annu. IEEE Semicond. Therm. Meas. Manag. Symp. (2008), pp. 26-31

30. U. S. Bhapkar, A. Srivastava, and A. Agrawal, Int. J. Therm. Sci. 74, 145 (2013)

31. U. S. Bhapkar, A. Srivastava, and A. Agrawal, Int. J. Heat Mass Transf. 79, 12 (2014)

32. L. D. Mangate and M. B. Chaudhari, Int. J. Therm. Sci. 89, 100 (2015)

33. M. Jabbal and J. Jeyalingam, Sensors Actuators, A Phys. 266, 273 (2017)

34. J. Jeyalingam and M. Jabbal, Sensors Actuators, A Phys. 280, 52 (2018)

35. E. Smyk, P. Gil, R. Gałek, and Ł. Przeszłowski, Actuators 9, 100 (2020)

36. E. Smyk, J. Wilk, and M. Markowicz, Appl. Sci. 11, 4600 (2021)

37. E. Smyk and M. Markowicz, Appl. Sci. 11, 652 (2021)

38. E. Smyk, Appl. Sci. 11, 9624 (2021)

39. P. Gil, E. Smyk, R. Gałek, and Ł. Przeszłowski, Int. J. Therm. Sci. 170, 107171 (2021)

40. M. Ikhlaq, M. Yasir, O. Ghaffari, and M. Arik, Exp. Heat Transf. (2021)

41. International Organization for Standardization (ISO), Acoustics - Determination of Sound Power Levels and Sound Energy Levels of Noise Sources Using Sound Pressure - Survey Method Using an Enveloping Measurement Surface over a Reflecting Plane (ISO 3746:2010) (ISO: Geneva, Switzerland, 2010), p. 48

42. C. K. W. Tarn and L. Auriault, 4th AIAA/CEAS Aeroacoustics Conf. 37, 880 (1998)

43. J. Kordík and Z. Trávníček, Exp. Therm. Fluid Sci. 86, 281 (2017)

44. H. Xia, P. G. Tucker, and S. Eastwood, Int. J. Heat Fluid Flow 30, 1067 (2009)

45. M. Sadeghian and M. G. Bandpy, J. Aeronaut. Aerosp. Eng. 9, (2020)

46. C. M. Crispo, C. S. Greco, and G. Cardone, Int. J. Heat Mass Transf. 126, 969 (2018)

47. C. M. Crispo, C. S. Greco, F. Avallone, and G. Cardone, Exp. Therm. Fluid Sci. 82, 136 (2017) 